\title{
Analytical one parameter method for PID motion controller settings
}

\author{
Johannes van Dijk, Ronald Aarts \\ University of Twente, dep. Mechanical Engineering, Automation and \\ Mechatronics Lab., Enschede, 7500AE Netherlands (e-mail: \\ j.vandijk@ctw.utwente.nl).
}

\begin{abstract}
In this paper analytical expressions for PID-controllers settings for electromechanical motion systems are presented. It will be shown that by an adequate frequency domain oriented parametrization, the parameters of a PID-controller are analytically dependent on one variable only, the cross-over frequency of the open loop transfer function. Analytical expressions are derived that relate the cross-over frequency clearly to the performance criteria for the closed loop system. In this paper the latter is shown in detail for servo problems. The effectiveness of the outlined approach is demonstrated by experimental results that were obtained from a two DOF tilting mirror system.
\end{abstract}

Keywords: Mechatronics, PID, Bode-design, frequency domain, MiMo-control, motion control

\section{INTRODUCTION}

The use of decentralized "classical" PID-control in electro mechanical systems, or mechatronic systems, is still widespread as it is a key component of industrial control due to its essential functionality and structural simplicity (Astrom and Hagglund (2001)). The literature on PID control design and tuning is quite extensive, see for an overview O'Dwyer (2009); Ang et al. (2005); Cominos and Munro (2002). The majority of papers is on tuning for process control applications. Only a few papers are on analytical frequency domain methods. As PID-control is strongly related to lead-lag compensation we refer to Wakeland (1976); Mitchell (1977); Wang (2003); Yeung et al. (1998); O'Dwyer (2007) for existing analytical frequency domain design procedures for lead-lag compensators.

However, none of the referred authors use the cross-over frequency ${ }^{1)}$ as the key parameter to tune or to set. Important in general control system design are performance and closed loop stability. For the design of electromechanical motion systems, performance is a low frequency issue and closed loop stability is a high frequency issue. This is commonly known as the mixed sensitivity problem. In this paper an approach is presented for the performance part of this problem. The approach described is original although it has some similarity with the two-step procedure described by Skogestad (2003) for process control. The approach is:

(1) Obtain a second order plant model and controller parametrization adequate for performance analysis.

(2) Determine the minimum cross-over frequency and PID-settings from the performance criteria.

In step 1 it is assumed that coupled Multi input Multi output (MiMo) systems are decoupled at some critical fre-

\footnotetext{
1) for the openloop transfer $L(s)$ yields at cross-over frequency , $\left|L\left(j \omega_{c}\right)\right|=1$
}

quencies using a (static) decoupling or interaction reducing strategy (Owens (1978); Boerlage et al. (2005)). Then one dimensional lumped parameter models representing the dominant system dynamics are adequate to support step 2 , the derivation of symbolic expressions between performance parameters and necessary cross-over frequency of the open-loop transfer function $L(s)=K(s) \cdot G_{0}(s)$, where $G_{0}(s)$ is the nominal model transfer function of the plant and $K(s)$ the transfer function of the controller. This derivation of symbolic expressions, to our opinion, is new. In step 2 high frequency dynamics due to parasitic modes are neglected. However, robust stability against these higher order dynamics restrict the cross-over frequency from above. The discussion on cross-over frequency and robust stability is considered to be outside the scope of this paper.

The background for the steps are described in the following sections, which are organized as follows. In section 2 , the necessary theoretical considerations to be used in the steps 1 and 2 are presented. A convenient parametrization of PID controllers will be presented based on the analytical frequency domain controller design procedures. Based on this parametrization it will be shown that performance requirements can be easily translated into a cross-over frequency specification given a general model of electrical mechanical systems. The approach presented is appropriate for motion systems subjected to a polynomial reference trajectory (Lambrechts et al. (2005)). Remark, that common considerations on performance in control engineering textbooks and in a large part of the PID-tuning publications (for references see O'Dwyer (2009)) are based on the step response. However, in practical motion systems a step is never applied as reference.

In section 3 the presented approach is demonstrated for a tilting mirror system. The determination of necessary cross-over frequency and PID-controller settings are outlined. Experimental results are obtained from a test set- 
up and show the effectiveness of the outlined approach. Section 4 summarizes the conclusions.

\section{FORMULATION OF ANALYTICAL EXPRESSIONS}

In the following subsections general model derivation, PID-controller parametrization and the analytical expressions between performance parameters and cross-over frequency will be addressed. It is assumed that performance is a low-frequency issue.

\subsection{Model derivation}

Decoupling The controller design for performance approach to be described is based on Single input Single output (SiSo) systems. It is also adequate for decoupled MiMo systems. Often MiMo systems suffer from interaction between inputs and outputs of the plant. In order to make the $i^{t h}$ output respond to the $i^{\text {th }}$ input alone and hence to reduce interaction it is assumed in the following that MiMo systems are decoupled by decoupling transformations. The decoupling transformations reduce the problem to the design of a number of non-interacting control loops.

First, the decoupling transformation matrices $(A, B)$ for the coupled MiMO system $G(s)$ are to be obtained, resulting in the diagonal plant $G_{d}(s)$ "seen" by the controller:

$$
G_{d}(s)=B G(s) A,
$$

of which the entries are SiSo transfer functions. In section 3 we apply a practically powerful static decoupling method. We use the decoupling strategy as described by Owens (Owens (1978)) that uses static real transformation matrices, which are known as the dyadic transfer function matrices. Second, the so-called decentralized controllers for the decoupled system have to be designed. Each of these controllers can be designed using methods for SiSo systems. For this purpose the SiSo plant is described next.

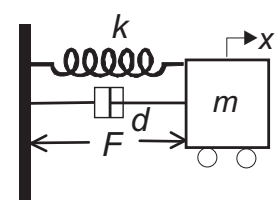

Fig. 1. General nominal model of motion system used for performance analyses/synthesis

The SiSo plant model Controlled electro-mechanical motion systems are often actuated by inductive actuators applied with either voltage- or current-mode power amplification. For control synthesis, considering performance, the mass of the subsystem to move is assumed rigid and a one dimensional model as shown in figure 1 will be adequate. This model is called "the nominal model" and is used for performance synthesis. In figure $1 k$ is the stiffness in actuated direction, $m$ is the mass to move, $F$ is the force supplied by the actuator and $d$ is the damping constant. From figure 1 the basic transfer function $G_{f}(s)$ between position $x$ of the mass and actuator-force $F$ can be obtained:

$$
G_{f}(s)=\frac{x(s)}{F(s)}=\frac{1}{m s^{2}+d s+k}
$$

In case of voltage control the force

$$
F=\frac{U \cdot k_{m}}{R}
$$

where $U$ is the applied voltage by the power amplifier, $k_{m}$ is the motor constant, and $R$ the resistance of the coil. In that case:

$$
d=\frac{k_{m}^{2}}{R}
$$

is the damping due to back-emf. Hence, in the case of voltage control (2) becomes:

$$
\frac{x(s)}{U(s)}=G_{u}(s)=\frac{\frac{k_{m}}{R \cdot m}}{s^{2}+\frac{k_{m}^{2}}{R \cdot m} s+\frac{k}{m}}
$$

In current mode control, $F=k_{m} \cdot i$ and the assumption $d=0$ is made. The current applied by the power amplifier is denoted by $i$ and (2) becomes:

$$
\frac{x(s)}{i(s)}=G_{i}(s)=\frac{\frac{k_{m}}{m}}{s^{2}+\frac{k}{m}}
$$

In case the mechanical subsystem does not have stiffness in the actuated direction $k=0$. In order to prevent hihg actuator power, the stiffness $k$ is required to be low. Therefore, the (undamped) resonance frequency in (5) and (6) $\omega_{1}=\sqrt{\frac{k}{m}}$ is low or zero. Typically, this resonance frequency is within the control bandwith. Consequently, near the cross-over frequency we can consider the high frequency $\left(\omega>\omega_{1}\right)$ approximation of (5) and (6) :

$$
\frac{x(s)}{U(s)}=G_{u, H F}(s)=\frac{c_{u}}{m s^{2}} \quad \forall \quad \omega>\omega_{1}
$$

where in (7) $c_{u}=k_{m} / R$. In the sequel we will use $m_{e q}$ for either $\frac{m}{c_{u}}$ or $\frac{m}{k_{m}}$, depending on the type of amplifier applied. So, both for voltage or current control (2) is written as:

$$
\frac{x(s)}{\operatorname{inp}(s)}=G_{n o m}(s)=\frac{\frac{1}{m_{e q}}}{s^{2}+\frac{d}{m} s+\frac{k}{m}}
$$

The high frequency (HF) approximation of (8) becomes:

$$
\frac{x(s)}{\operatorname{inp}(s)}=G_{H F}(s)=\frac{1}{m_{e q} s^{2}} \quad \forall \quad \omega>\omega_{1}
$$

\subsection{PID controllers}

The usual parallel form of an industrial PID-controller is:

$$
K(s)=K_{p}+\frac{K_{i}}{s}+\frac{K_{d} s}{s \tau+1}
$$

where $K_{p}, K_{i}$, and $K_{d}$ are the proportional, integral and derivative gains respectively. $\tau$ is an a priori selected timeconstant which limits the high frequency gain of the PIDcontroller. For the purpose of frequency domain loopshaping (10) is rewritten in the series form

$$
K(s)=k_{p} \cdot \frac{\left(s \tau_{z}+1\right)\left(s \tau_{i}+1\right)}{s \tau_{i}\left(s \tau_{p}+1\right)}
$$

where the parameters $k_{p}, \tau_{z}, \tau_{i}$ and $\tau_{p}$ are uniquely related to $K_{p}, K_{i}, K_{d}$ and $\tau$ (Grassi et al. (2001)). Characteristic for the PID-controller is its high gain at low-frequencies. Figure 2 shows the bode-diagram of this PID-controller. The first corner-point ${ }^{2}$ ) is determined by the transferzero in $\frac{1}{\tau_{i}}$, the second corner-point is determined by the

\footnotetext{
2) The point where the asymptote is turning into a line with different angle. The corresponding frequency is called corner-frequency.
} 


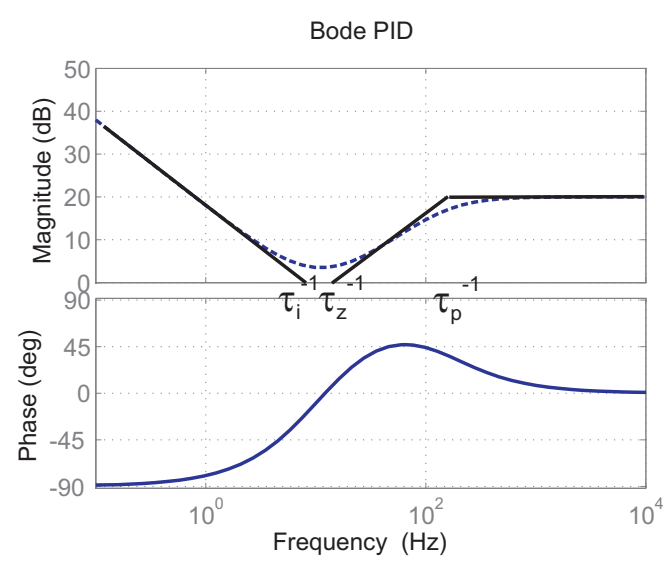

Fig. 2. Bode diagram of PID-controller

transfer-zero in $\frac{1}{\tau_{z}}$. Finally, the gain at high frequency is limited by the corner-point due to the pole in $\frac{1}{\tau_{p}}$. The corner-frequency $\frac{1}{\tau_{z}}$ indicates where the derivative action is started. Since the phase-lag of the I-action should not interfere with phase-lead of the derivative action, the corner-frequency $\frac{1}{\tau_{i}}$, indicating the stop of the integralaction, should be lower in frequency than the start of the derivative action. Therefore, it is chosen $\tau_{i}=\beta \cdot \tau_{z}$, $\beta>1$. Due to the zero of the transfer in $\frac{1}{\tau_{z}}$, the controller provides phase-lead in a certain frequency range, as shown in figure 2. Typically, the PID-controller is used to increase the cross-over frequency and to provide sufficient phasemargin at the cross-over frequency.

At first analytical expressions for the parameters of the PID-controller as a function of the cross-over frequency $\omega_{c}$, are derived. As outlined $\tau_{i}=\beta \cdot \tau_{z}$, and furthermore $\tau_{p}$ in (11) is replaced by $\alpha \cdot \tau_{z}$. The amount of phase-lead is determined by $\alpha$. Usually, this parameter is set between 0.1 to 0.3 to provide a desired amount of phase lead.

From figure 2 we observe that the position of the second corner-point of the asymptote is determined by the location of the zero at $\frac{1}{\tau_{z}}$. The position of the high frequency corner-point is determined by the location of the pole at $\frac{1}{\tau_{p}}$. The maximum phase-lead is obtained at the frequency $\omega_{p h m}$, which is the geometrical mean of the corner points:

$$
\omega_{p h m}=\log ^{-1} \frac{\log \frac{1}{\tau_{z}}+\log \frac{1}{\tau_{p}}}{2}=\sqrt{\frac{1}{\tau_{z} \cdot \tau_{p}}}
$$

By using the asymptotes the gain of the PID-controller at the frequency where the maximum phase-lead is obtained reads as:

$$
\left|K\left(j \omega_{p h m}\right)\right|=k_{p}\left(\sqrt{\frac{1}{\alpha}}\right)
$$

The maximum phase-lead is at $\omega=\omega_{p h m}$ and the phase of the plant at frequencies well above $\omega_{1}$ is $-180^{\circ}$. As a consequence, to use the phase-lead of the PID-controller most effectively we should design it in such a way that $\omega_{p h m}$ is located at the desired cross-over frequency:

$$
\omega_{c}=\omega_{p h m}
$$

At the cross-over frequency $\left|K G_{H F}\right|=1$, so

$$
\left|K \cdot G_{H F}\right|_{\omega_{c}}=\left|\frac{K\left(j \omega_{c}\right)}{m_{e q} \cdot\left(j \omega_{c}\right)^{2}}\right|=\frac{k_{p} \sqrt{\frac{1}{\alpha}}}{m_{e q} \omega_{c}^{2}}
$$

Using (12) (14) and (15) the following relations between $\tau_{z}, \tau_{i}, \tau_{p}, k_{p}$ and $\omega_{c}$ can then be obtained:

$$
\begin{aligned}
& \tau_{z}=\frac{\sqrt{\frac{1}{\alpha}}}{\omega_{c}} \quad, \tau_{i}=\beta \cdot \tau_{z} \\
& \tau_{p}=\frac{1}{\omega_{c} \cdot \sqrt{\frac{1}{\alpha}}}, k_{p}=\frac{m_{e q} \omega_{c}^{2}}{\sqrt{\frac{1}{\alpha}}}
\end{aligned}
$$

Eq. (16) gives the analytical expression for the parameters of a PID-controller as a function of the cross-over frequency. In practice the PID-controller is extended with a low-pass filter to prevent amplification of noise and possibly with notch filters to cope with parasitic dynamics.

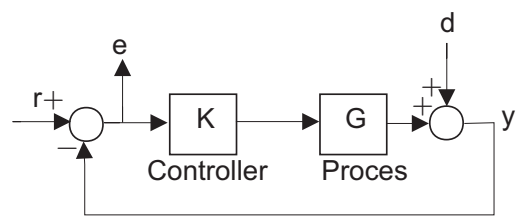

Fig. 3. Block-diagram of controlled system

\subsection{Performance requirement and cross-over frequency}

Figure 3 shows the block-diagram of the controlled system, where reference $r$ and disturbance $d$ are inputs and the servo-error $e$ is an output. The closed-loop transfer $S(s)$ between $r$ and $e$ and between $d$ and $e$ (when omitting the minus sign), is called Sensitivity function and can be written as:

$S(s)=\frac{s\left(s \tau_{p}+1\right)\left(s^{2}+\frac{d}{m} s+\frac{k}{m}\right)}{s\left(s \tau_{p}+1\right)\left(s^{2}+\frac{d}{m} s+\frac{k}{m}\right)+\frac{k_{p}}{m_{e q} \tau_{i}}\left(s \tau_{z}+1\right)\left(s \tau_{i}+1\right)}$

where we have substituted for $K(s)$ the transfer function of the controller (11) and for $G(s)$ the transfer function of the plant (8).

In the following we will address the dynamic system behavior of these systems in case the reference is described in time as a third degree polynomial. The reference ( $r$ in figure 3 ) is described by:

$$
\begin{aligned}
& r(t)=\frac{16}{3} \cdot h_{m}\left(\frac{t}{t_{m}}\right)^{3}, \quad 0 \leq t \leq \frac{t_{m}}{4} \\
& r(t)=\frac{32 h_{m}}{t_{m}^{3}}\left(\frac{t_{m} t^{2}}{4}-\frac{t^{3}}{6}-\frac{t_{m}^{2} t}{16}+\frac{t_{m}^{3}}{192}\right), \frac{t_{m}}{4}<t \leq \frac{3 t_{m}}{4} \\
& r(t)=\frac{32 h_{m}}{t_{m}^{3}}\left(\frac{-t_{m} t^{2}}{2}+\frac{t^{3}}{6}+\frac{t_{m}^{2} t}{2}-\frac{13 t_{m}^{3}}{96}\right), \frac{3 t_{m}}{4}<t \leq t_{m} \\
& r(t)=h_{m}, \quad t>t_{m}
\end{aligned}
$$

where $h_{m}$ is the total displacement of the trajectory, or set-point and $t_{m}$ is the time available for the trajectory, or setup-time.

A prediction of the error $e$ can be obtained from the fact that the error behavior is the result of filtering the reference $r$ by $S(s)$. The static gain of the filter $S$ scales the (dominant) components of the reference into 
the servo-error and the filter introduces delay. Second, the components of the reference which play a dominant role in the error behavior must be identified. The power density of the reference will have a peak at

$$
\frac{2 \pi}{t_{m}}=\omega_{d} .
$$

The power density will be significant for frequencies $\omega<$ $\omega_{d}$, but will decrease rapidly for $\omega>\omega_{d}$. For sufficient fast tracking of the reference we should assure $\omega_{d}<<\omega_{c}$ and hence the reference is considered as a low frequency disturbance on the system. Therefore, the low-frequency behavior of $S$ can be considered. For that reason we can simplify $S(s)$ to the so called low frequency equivalent $S_{L F}(s)$ where only components with a frequency content smaller than the cross-over frequency $\omega_{c}$ are taken into account. For the fourth order denominator of $S(s)$ the following can be stated. $S(s)$ will have a complex conjugated pole-pair with the frequency of the cross-over frequency $\omega_{c}$. These are not of interest when considering low frequency behavior of $S$. Also there will be a relatively high frequency real valued pole around $-\frac{1}{\tau_{p}}=-\sqrt{1 / \alpha} \cdot \omega_{c}$, which is neither of interest. As a consequence, there is one real valued pole which location needs attention. It is expected that this pole is in the low frequency region. For that reason the denominator of $S(s)$ is simplified to be of first order in order to approximate the location of the pole.

Considering simplification of the numerator of $S(s)$ the following can be observed. $S(s)$ will have zeros corresponding with the poles of the plant and the controller. So, the zero in 0 and the zeros corresponding with the low frequency plant poles need to be taken into account. The high frequency zero due to the controller pole in $-\frac{1}{\tau_{p}}$ is neglected. Hence, (17) can be approximated by:

$$
S_{L F}=\frac{s^{3}+\frac{d}{m} s^{2}+\frac{k}{m} s}{\left(\frac{k}{m}+\left(\tau_{i}+\tau_{z}\right) \cdot \frac{k_{p}}{m_{e q} \cdot \tau_{i}}\right) s+\frac{k_{p}}{m_{e q} \cdot \tau_{i}}} .
$$

Substitution of $\frac{k}{m}=\omega_{1}^{2}$ and (16) into (21) results in:

$$
S_{L F}=\frac{s^{3}+\frac{d}{m} s^{2}+\omega_{1}^{2} s}{\left(\omega_{1}^{2}+\left(1+\frac{1}{\beta}\right) \sqrt{\alpha} \omega_{c}^{2}\right) s+\frac{1}{\beta} \alpha \omega_{c}^{3}} .
$$

Expression (21) can be used to predict the maximum servo error, the set-point error and the amount of disturbance rejection in relation with a required cross-over frequency.

\section{Prediction of the maximum servo error as a function of $\omega_{c}$}

In order to predict the maximum servo error, the static gain or scaling factor of $|S(0)|$ must be derived, the delay is of no concern. Therefore, the denominator of (21) can be further simplified to the zero-order approximation. Then, the low frequency approximation of the servo-error $e_{L F}(s)$ is written in the Laplace domain as:

$$
e_{L F}(s)=\frac{s^{3}+\frac{d}{m} s^{2}+\omega_{1}^{2} s}{\frac{1}{\beta} \alpha \omega_{c}^{3}} \cdot r(s)
$$

and in the time domain as:

where

$$
e_{L F}(t)=k_{j} \cdot \dddot{r}(t)+k_{a} \cdot \ddot{r}(t)+k_{v} \cdot \dot{r}(t)
$$

$$
k_{j}=\beta \frac{1}{\alpha \cdot \omega_{c}^{3}}, \quad k_{a}=\beta \frac{\frac{d}{m}}{\alpha \cdot \omega_{c}^{3}}, \quad k_{v}=\beta \frac{\omega_{1}^{2}}{\alpha \cdot \omega_{c}^{3}}
$$

As is shown by (23) the low-frequency approximation of the servo-error $e_{L F}$ will depend on the jerk $\dddot{r}(t)$, the acceleration $\ddot{r}(t)$ and the velocity $\dot{r}(t)$ profiles of the reference, the model parameters $d, m$ and $\omega_{1}=\sqrt{k / m}$ as well as on the control parameters which are expressed in the cross-over frequency $\omega_{c}$ and the parameters $\alpha$ and $\beta$ using (16).

Figure 4 shows a simulation result of an error response and the different components of the prediction of the error using (23) for an example system. As reference a third degree trajectory is used as described by (18).

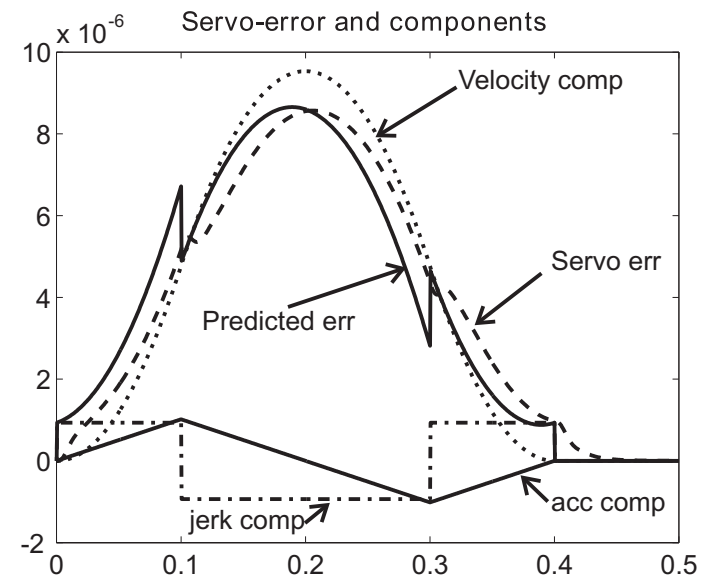

Fig. 4. Simulation results of servo-error and its components according to (23). Simulation are obtained with plant (8) parameters: $k=100 \mathrm{~N} / \mathrm{m}, m=0.0979 \mathrm{~kg}$, $k_{m}=3.2 \mathrm{~N} / \mathrm{A}, R=10 \Omega m_{e q}=m / c_{u}$. Controller parameters: $\omega_{c}=60 \mathrm{~Hz} ., \alpha=0.2, \beta=2$. Reference parameters: $h_{m}=0.01 \mathrm{~m}, t_{m}=0.4 \mathrm{~s}$

The time derivatives of this reference can be computed easily and their contributions to the servo-error (23) are shown separately. Some remarks can be made after observing the servo-error. The maximum of the predicted servo error arises at $\approx \frac{t_{m}}{2}$. At $\frac{t_{m}}{2}$ both the jerk $\dddot{r}$ and the velocity $\dot{r}$ have their maximum values $j_{\max }$ and $v_{\max }$ respectively. The simulated servo-error (dashed-line) is shifted in time compared to the sum of the components of the prediction (23) (the solid-line). The prediction overestimates the maximum of the servo-error only slightly

In case a current amplifier is applied, which is usual, $d=0$ and the acceleration-term has zero contribution. In case a voltage amplifier is used the acceleration has neither a contribution in the prediction of the maximum servo-error since $\ddot{r}\left(\frac{t_{m}}{2}\right)=0$. Apparently, the second term in the error prediction (23) can be ignored in the estimation of the maximum error:

$e_{L F}\left(\frac{t_{m}}{2}\right)=\beta \frac{j_{\max }}{\alpha \cdot \omega_{c}^{3}}+\beta \frac{\omega_{1}^{2} \cdot v_{\max }}{\alpha \cdot \omega_{c}^{3}}=\frac{2 \beta h_{m}}{\alpha \omega_{c}^{3} t_{m}}\left(-\frac{16}{t_{m}^{2}}+\omega_{1}^{2}\right)$

In case the stiffness $k=0$ or $\omega_{1}$ is small, the jerk-term dominates in (25). In case $\omega_{1}>\frac{4}{t_{m}}$ and taking only the velocity term into account results in a slight over estimation of the the maximum servo-error . Therefore, two distinct relations can be derived from (25) for computation of the 


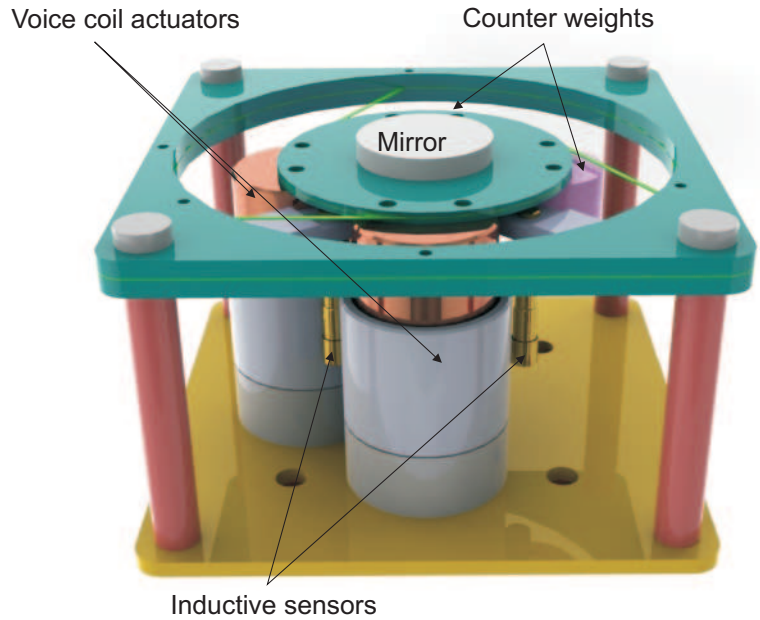

Fig. 5. Geometry of prototype tilting mirror

cross-over frequency in case the maximum servo-error is specified as $e_{L F}\left(\frac{t_{m}}{2}\right)=e_{\max }$, and (18) is used as reference:

$$
\begin{array}{r}
0 \leq \omega_{1}<\frac{4}{t_{m}} \omega_{c}=\sqrt[3]{\frac{\beta \cdot j_{\max }}{\alpha \cdot e_{\max }}}=\sqrt[3]{\frac{32 \beta h_{m}}{\alpha e_{\max } t_{m}^{3}}} \\
\omega_{1} \geq \frac{4}{t_{m}} \omega_{c}=\sqrt[3]{\frac{\beta \omega_{1}^{2} \cdot v_{\max }}{\alpha \cdot e_{\max }}}=\sqrt[3]{\frac{2 \beta \omega_{1}^{2} h_{m}}{\alpha e_{\max } t_{m}}}
\end{array}
$$

Often in mechatronic systems a feedforward is added to increase the performance. This feedforward can easily be included in the relations between error and reference. However, this is beyond the scope of this paper.

\section{CONTROLLER DESIGN FOR A TILTING MIRROR SET-UP}

A two input two output example from laser surface treatment will be used as a case study. Figure 5 shows a prototype of a 2 degrees of freedom (DOF) tilting mirror mechanism. The mirror manipulates the laser spot in a plane (two degrees of freedom). The prototype consists of a mirror-plate with a simple mirror mounted on it's surface. The mirror plate is suspended by four wire springs. These wire springs suppress, in principle, 4 of the 6 DOF of the mirror-plate (Soemers (2009)). The 2 DOF's left are rotation around $\mathrm{x}$-axis and $\mathrm{y}$-axis. These rotations (tilts) will be accomplished by voicecoil motors (VCM's) as indicated in figure 5. Counterweights are used to balance the coil masses of the VCM's connected to the mirror plate, with the goal to obtain a symmetric dynamic problem. Inductive sensors are mounted in order to measure the mirror-plate displacement.

3.1 Determination of cross-over frequency from performance requirements

In order to test the above outlined theory, the tilting mirror mechanism is subjected to a reference trajectory as described by (18). The trajectory is such that at sensor 1 a displacement of the mirror plate of $h_{m}=0.5 \mathrm{~mm}$ is demanded while sensor 2 should measure zero displacement difference. Remark that this requires actuation of

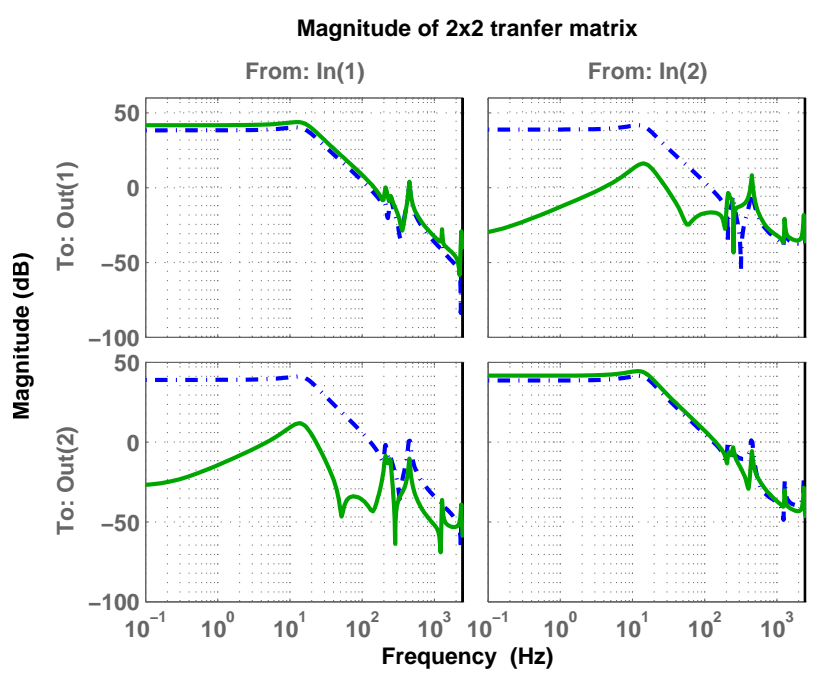

Fig. 6. Bode magnitude plot of transfer-matrix, identified plant (dotted) and decouple identified plant (solid)

both actuators. During this motion the maximum servoerror is $e_{\max }=10 \mu$. The set-up time $t_{m}=0.1 \mathrm{sec}$.

Obtaining the model Identification techniques (Overschee van and de Moor (1996) ) are used in order to obtain a model from the set-up. For this reason the actuator inputs $\widetilde{\boldsymbol{u}}(k)$ are excited with a white Gaussian noise sequence and the sensor outputs $\boldsymbol{y}(k)$ are recorded.

To validate the quality of the model, the VarianceAccounted-For (VAF) Overschee van and de Moor (1996) measure is used. A $2 \times 2$ transfer model of order 16 was identified, which gave a VAF of $99.67 \%$ on the validation data. Based on this VAF value, it can be stated the model describes the system well enough. The Bode magnitude plot of the result of the $2 \times 2$ state space obtained by identification is shown in figure 6 .

Next to the identified transfer-matrix the bode-magnitude results of decoupling or plotted in figure 6 . From which one can conclude that the controller indeed 'sees' an almost diagonal plant in the frequency region of interest from 0.1 to $200 \mathrm{~Hz}$. Note that in (1) the static transfer-matrix $B$ is a rotation of the frame that spans the sensor space to the frame of the controller space and $A$ is responsible for rotating the controller space frame to actuator space frame.

Desired cross-over frequency and controller settings The bodeplots show that resonance frequencies of the tiltmodes $\omega_{1}$ are approximately $14.5 \mathrm{~Hz}$.. As a consequence $\omega_{1}>\frac{4}{t_{m}}$ and therefore we can use (27) to compute the desired cross-over frequency. The result is $\omega_{c}=70 \mathrm{~Hz}$, with $\alpha=0.2, \beta=2$. The product of the parameters $m_{e q_{1}} \cdot \omega_{c}^{2}$ and $m_{e q_{2}} \cdot \omega_{c}^{2}$, needed for the proportional gains of the two controllers according to (16), can be obtained from the evaluation of the diagonalized plant gains at the desired cross-over frequency $K_{g}$ :

$$
m_{e q_{i}} \cdot \omega_{c}^{2}=\operatorname{Re}\left(-K_{g_{i, i}}\right)^{-1} \quad i=1 . .2
$$

Where $R e$ delivers the real parts of the complex values of $K_{g}^{-1}$. The evaluation of an electromechanical plant at 


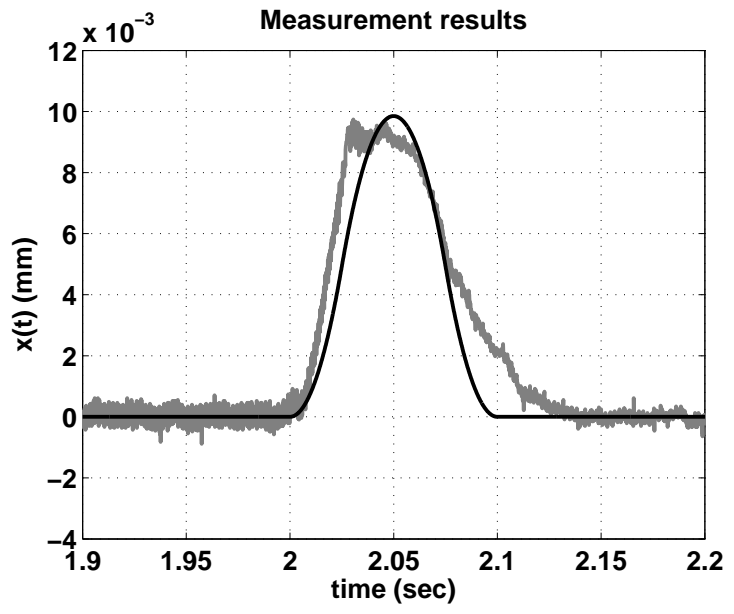

Fig. 7. Error between reference 1 and sensor-output 1 , black line predicted error $e_{\text {pred }}=k_{v} \dot{r}(t)$ according to (23), with only the velocity part. Grey line is measured error. Sensor KD2446-5CM res. $<0.5 \mu m$.

cross-over frequency usually results in a real matrix, since the phase at cross-over frequency will be -180 degrees. In case the phase differs from -180 degrees it is suggested to

use the ALIGN algorithm (MacFarlane and Kouvaritakis (1977)) to obtain the best real-approximation of the complex matrix. According to (16) all parameters of the two PID-controllers are known. The diagonal PID-controller is discretized using a sample frequency of $8333 \mathrm{~Hz}$ and implemented on the set-up hardware.

Figure 7 shows the servo error-response after applying the references as described on the controlled setup. Figure 7 also shows the predicted error $e_{\text {pred }}=k_{v} \dot{r}(t)$ according to (23), with only the velocity part taken into account. Considering the maximum servo-error the resemblance between prediction and measured maximum servo-error is large. From which it is concluded that the outlined approach is very promising in the determination of minimum cross-over frequencies for servo-problems.

\section{CONCLUSIONS}

Shown are analytical expressions between de three PIDcontroller parameters and the desired cross-over frequency, in the case of motion systems. Moreover it has been shown that the cross-over frequency can be expressed in the performance parameters of motion systems, in detail it is shown for a servo-problem. The reference and with that the performance parameters, in this class of problems are usually formulated as a trajectory where $h_{m}$ is the total displacement of the trajectory, or set-point and $t_{m}$ is the time available for the trajectory, or setup-time and $e_{\max }$ the maximum allowable servo-error. The relation between maximum servo-error and cross-over frequency is then:

$$
e_{\max }=\frac{2 \beta h_{m}}{\alpha \omega_{c}^{3} t_{m}}\left(-\frac{16}{t_{m}^{2}}+\omega_{1}^{2}\right)
$$

where $\omega_{1}$ is the first resonance frequency which is set to zero in case of a motion system consisting of a moving mass without stiffness in actuated direction. The PID-controller settings are:

$$
\begin{gathered}
\tau_{z}=\frac{\sqrt{\frac{1}{\alpha}}}{\omega_{c}} \quad, \tau_{i}=\beta \cdot \tau_{z} \\
\tau_{p}=\frac{1}{\omega_{c} \cdot \sqrt{\frac{1}{\alpha}}}, k_{p}=\frac{m_{e q} \omega_{c}^{2}}{\sqrt{\frac{1}{\alpha}}}
\end{gathered}
$$

where $\alpha$ is usually 0.1 or 0.2 and $\beta>1$.

\section{REFERENCES}

Ang, K.H., Chong, G., and Li, Y. (2005). "PID control system analysis, design, and technology". Control Systems Technology, IEEE Transactions on, 13(4), 559 576.

Astrom, K.J. and Hagglund, T. (2001). "The future of PID control". Control Engineering Practice, 9(11), pp. 1163-1175.

Boerlage, M., Steinbuch, M., and Angelis, G. (2005). Frequency reponse based multivariable control design for motion systems. In IEEE Conference on Control Applications, CCCA 2005, Toronto.

Cominos, P. and Munro, N. (2002). "PID controllers: recent tuning methods and design to specification". IEE Control Theory and Applications, 149(1), 46 -53.

Grassi, E., Tsakalis, K., Dash, S., Gaikwad, S., MacArthur, W., and Stein, G. (2001). "Integrated system identification and PID controller tuning by frequency loopshaping". Control Systems Technology, IEEE Transactions on, 9(2), 285 -294. doi:10.1109/87.911380.

Lambrechts, P., Boerlage, M., and Steinbuch, M. (2005). Trajectory planning and feedforward design for electromechanical motion systems. Control Engineering Practice, 13(2), 145 - 157.

MacFarlane, A. and Kouvaritakis, B. (1977). A design technique for linear multivariable feedback systems. International Journal of Control, 25(6), 837-874.

Mitchell, J.R. (1977). "Comments on Bode Compensator Design". Transaction on Automatic Control, 22(10), pp. 869-870.

O'Dwyer, A. (2007). "Development of analytical design methods for single stage phase lead and phase lag compensators". In ISSC sept 13-14, 2007, Derry, 133-137.

O'Dwyer, A. (2009). Handbook of PI and PID controller tunig rules. Imperial College Press, London, third edition edition.

Overschee van, P. and de Moor, B. (1996). Subspace Identification for Linear Systems: Theory - Implementation - Applications. Springer. ISBN 0-7923-9717-7.

Owens, D. (1978). Feedback and Multivariable Systems. Peter Peregrinus, Stevenage., Southgate House.

Skogestad, S. (2003). "Simple analytical rules for model reduction and PID controller tuning". Journal of Process Control, 13, 291-309.

Soemers, H. (2009). Design principles for precision mechanisms. T-Point Print VoF, Enschede. ISBN:9789036531030

Wakeland, W.R. (1976). "Bode Compensator Design". Transaction on Automatic Control, 21(10), pp. 771-773.

Wang, F.Y. (2003). "The exact and unique soluition for phase-lead and phase-lag compenstion". IEEE Transaction on Education, 46(2), pp. 258-262.

Yeung, S.K., Wong, K., and Chen, K. (1998). "A nontrial and error method for lag-lead compensator design". IEEE Transaction on Education, 41(1), pp. 76-80. 